\title{
LOW FREQUENCY ELECTROMAGNETIC WAVES INCREASE HUMAN SPERM MOTILITY - A PILOT STUDY REVEALING THE POTENT EFFECT OF 43 kHz RADIATION
}

\author{
${ }^{1}$ Medical University (Collegium Maximum), Lublin, Poland \\ Faculty of Health Sciences, Diagnostic Techniques Unit \\ ${ }^{2}$ Lublin University of Technology, Lublin, Poland \\ Institute of Electrical Engineering and Electrotechnologies \\ ${ }^{3}$ Institute of Rural Health, Lublin, Poland \\ Department for Health Problems of Aging
}

ARTUR WDOWIAK ${ }^{1}$, PAWEL A. MAZUREK ${ }^{2}$, ANITA WDOWIAK ${ }^{1}$, and IWONA BOJAR ${ }^{3}$

\begin{abstract}
Objectives: Abnormalities in the timing and course of spermatozoa capacitation and hyperactivation underlie common pathologies related to male infertility. Recent data shows that low frequency electromagnetic waves may influence cell membrane potential and permeability. It is therefore possible that low frequency electromagnetic waves could affect the maturation and motility processes of spermatozoa. The $43-\mathrm{kHz}$ wave generator was used for modeling the impact of environmental exposure to low frequency electromagnetic radiation on human sperm. Material and Methods: Sperm samples were gathered from 103 fertile, healthy men aged 25-30 years old and performed computer-assisted sperm analysis. After initial examination, each participant's semen sample was divided into 2 aliquots (control and experimental) and placed in separate automated incubators. The samples constituting the experimental group were placed into the exposure system that emitted 43-kHz electromagnetic waves. Sperm motility was assessed at $3 \mathrm{~h}, 12 \mathrm{~h}$ and $24 \mathrm{~h}$. Results: Exposure to a 43-kHz radio frequency increased the percentage of sperm in progressive motility by up to $5.8 \%$ and the velocity of said sperm by up to $2 \mu \mathrm{m} / \mathrm{s}$. Moreover, the total number of hyperactivated spermatozoa was significantly increased in the semen exposed to the electromagnetic signal. Conclusions: In vivo environmental exposure to $43-\mathrm{kHz}$ waves may promote the development of infertility related to premature capacitation outside of the vaginal tract. Exposing semen to this particular frequency may also boost the capacitation and hyperactivation of spermatozoa in vitro, prior to conducting assisted reproductive therapies. Int J Occup Med Environ Health 2018;31(6):723-739
\end{abstract}

Key words:

Electromagnetic radiation, Semen motility, Sperm hyperactivation, Sperm kinetic variables, Metal detecting, Infertility

Funding: this study was supported by International Scientific Association for the Support and Development of Medical Technologies (grant No. 8B/2015 entitled "Evaluation of the influence of electromagnetic fields on the parameters of male semen," grant manager: Artur Wdowiak).

Received: September 7, 2017. Accepted: January 4, 2018.

Corresponding author: Artur Wdowiak, Medical University (Collegium Maximum), Faculty of Health Sciences, Diagnostic Techniques Unit, Staszica 4-6, 20-081 Lublin, Poland (e-mail: wdowiakartur@gmail.com). 


\section{INTRODUCTION}

In order to be able to fertilize the ovum, human sperm must undergo capacitation - a series of biochemical changes mainly related to rearrangement of the cell membrane. Capacitation occurs after the sperm is introduced to the environment of the vaginal tract, and is followed by modification of the path and motility of gametes. During the ascent, spermatozoa tail motility increases in amplitude and curvature, generating a greater force of movement. As a result, the sperm enters the so-called hypermotility state, and it is this state which allows for a curved path of travel, leading to the escape from the epithelial cells of the fallopian tube, subsequent penetration of the zona pellucida, and ultimately fertilization of the ovum. It is this combined force and trajectory of travel that is the point of difference between mature and premature sperm; the latter is only able to move in a straight line and therefore unable to fertilize the oocyte [1]. Interestingly, premature hyperactivation occurring in the seminal plasma results in energy depletion of the sperm, which subsequently becomes unable to fertilize the oocyte. The maintenance of balance within the oxidoreductase system is essential for capacitation to be triggered at precisely the right moment. Shifting this balance in one direction causes damage to sperm, and a shift in the opposite direction could trigger premature capacitation [2]. Recent data suggests that particular frequencies of electromagnetic radiation (EMR) are able to influence the time and course of capacitation. Herein, the impact of low frequency EMR (LF-EMR) on the motility and condition of human spermatozoa has been studied.

Electromagnetic radiation is a distortion of the electromagnetic field (EMF) that extends indefinitely throughout space. Electrical and magnetic components of radiation mutually induce one another, creating varying EMFs. Electromagnetic signals emitted by both natural and artificial sources are thought to have some effect on human health, and although this kind of energy may affect tissue function in various ways, this phenomenon is not yet fully understood. It is expected that interactions between EMR and a living organism may depend on the amount and the form of transferred energy, as well as the type of tissue [3]. The tissues constituting the human reproductive system are known to be very sensitive to various external factors but the effects of EMR on this system are ambiguous [4]. The impact of EMR on the male reproductive tissues may include thermal effects, the generation of oxidative stress as well as the appearance of additional potentials across cellular membranes resulting in altered ion transport and membrane protein conformation [5,6]. Such effects are observed for fields of several hundred millivolts (much higher than the resting voltages across membranes of highly polarized organelles such as mitochondria) [7]. When a current is applied across a tissue, most of the voltage drop appears across the membrane at LFs; the reason this occurs only at LFs is because at high (gigahertz) frequencies the capacitance of the membrane effectively shorts out the membrane resistance. The change in voltage may be described by the ER equation, where $\mathrm{E}$ is external field and $\mathrm{R}$ is cell radius in the direction parallel to the field [8]. Radio-frequency (RF)-induced changes in membrane potential can reach up to $100 \mathrm{mV}$.

Differences in composition cause particular proteins to behave differently when exposed to EMR [9]. More specifically, proteins consist of many chains of amino acids, some of which are polar; this polarity causes these amino acids to behave differently to neutral amino acids when exposed to EMFs. High irradiation levels are also known to affect protein folding [10]. Proteins are often complexed and display electrostatic contact; as such, exposure to RF-EMR may alter the organization of a large amount of proteins within the cell. It may also affect conformation of particular proteins, aggregation of homo- and heteromeres, and their stability [10].

Many recent studies on the influence of EMR on male fertility concern the bands associated with mobile telephony [4]. The detrimental impact of EMR is generally classified into thermal and non-thermal effects. The effects on 
sperm motility, density, DNA fragmentation and percentage of normal spermatozoa have been evaluated in a number of studies but the results are inconclusive. Their detrimental effects are explained by means of oxidative stress generation and the influence on the work of voltage-gated channel Hv1, which is the sensor of the electrical voltage responsible for the transport of calcium $\left(\mathrm{Ca}^{2+}\right)$ ions to the cell [8]. These disorders lead to cell growth inhibition, protein misfolding, and DNA breaks. Although the influence of EMR associated with metal detection has not been investigated thus far, one can expect a similar effect of these frequencies on male fertility.

The last 2 decades have seen the dynamic development of technologies able to transfer energy at LF (30-300 kHz) and very low frequency (VLF) (3-30 kHz). Such frequencies are used in devices enabling the detection of metal, or other objects, from deep within the earth. Hobby and industrial metal detectors, or georadars, have become objects of everyday use, and metal detector gates are used daily at airports and other public places. The increasingly advanced electrotechnologies and the increasing number of devices used in everyday life mean it is now necessary to understand the impact of waves emitted by such devices on humans [11]. In a previous study, the frequencies of electromagnetic waves usually emitted by metal detectors were identified $[12,13]$; in this study the impact of this frequency, specifically $43 \mathrm{kHz}$, on selected parameters of sperm motility has been assessed using the computer-aided sperm analysis (CASA) system.

\section{MATERIAL AND METHODS}

\section{Participants}

This study was conducted in 2015 at the Non-Public Health Care Unit "Ovum" of Reproduction and Andrology in Lublin. One hundred and three healthy, fertile men, aged 25-30 years old, were enrolled in the study. Participant fertility was confirmed by each man having already fathered at least one child, and health status was confirmed by medical examination. Smokers, men with clinically diagnosed features of reproductive organ inflammation and symptoms of systemic disease, and those with body weight disorders (i.e., with a body mass index $<17$ or $>30$ ) were excluded. Prior to enrollment, all participants signed a written consent form allowing the use of the donated biological samples and medical data for research purposes. The study was approved by the Ethics Committee at the Institute of Rural Medicine in Lublin (No. 05/2015).

\section{Sperm donation and computer-assisted sperm analysis}

Sperm was obtained by masturbation and examined directly after liquefaction according to criteria established by the World Health Organization (WHO) [14], using the computer-assisted sperm analysis (CASA) system SCA ${ }^{\circledast}$ Evolution. Prior to sperm donation, participants were required to abstain from sex and alcohol for 4 days.

The following analyses were calculated using the CASA system:

- motility analysis - characteristic of sperm motion in \%: fast progressive (type a), slow progressive (type b);

- distribution of the spermatozoa velocity - shared value in \% for groups of rapid, medium, slow and static;

- average values of velocity parameters

- curvilinear velocity (VCL) in $\mu \mathrm{m} / \mathrm{s}$ - a measure of the total distance traveled by a given sperm divided by the time elapsed (Figure 1);

- straight-line velocity (VSL) in $\mu \mathrm{m} / \mathrm{s}$ - the straight line distance from beginning to end of a sperm track divided by the time taken (Figure 1);

- average path velocity (VAP) in $\mu \mathrm{m} / \mathrm{s}$ - the average path velocity of sperm (Figure 1);

- linearity (LIN) in \% - the linearity of the curvilinear trajectory, VSL/VCL;

- straightness (STR) in \% - linearity of the spatial average path, VSL/VAP;

- wobble (WOB) in \% - measure of oscillation of actual trajectory about its spatial average path; 


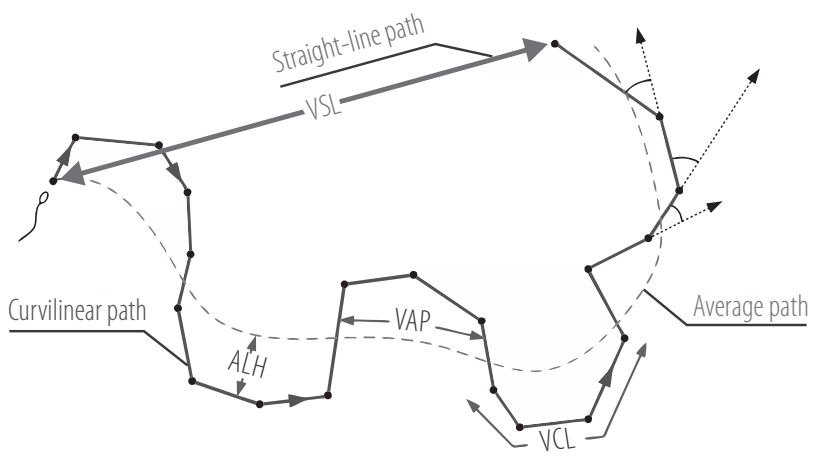

VSL - straight-line velocity; VCL - curvilinear velocity; VAP - average path velocity; ALH - lateral head displacement.

Fig. 1. Reference parameters of the spermatozoa motility track

- according to the low VAP cut-off and medium VAP cut-off, the sperm population was additionally divided into 4 categories: total, rapid, medium and slow;

- average values of other parameters:

- amplitude of lateral head displacement (ALH) in $\mu \mathrm{m}$;

- beat cross frequency (BCF) in $\mathrm{Hz}$;

- hyperactive total - the concentration of hyperactive sperm in total ejaculate in million.

\section{Hyaluronan binding assay}

A hyaluronan binding assay (HBA) kit (Biocoat, Washington, PA, USA) was used according to the manufacturer's instructions. In short, $10 \mu \mathrm{l}$ of sperm was placed into a chamber with a molecular layer of hyaluronan covalently linked to it, and covered with a special transparent Cell-Vu grid coverslip. After 10 min at room temperature, bound sperm was attached to hyaluronic acid (HA) in a "head-first" orientation, and unbound sperm was moving freely within the chamber.

\section{DNA fragmentation index}

In order to determine the percentage share of fragmented DNA in sperm, a sperm chromatin dispersion test (SCD) was used, according to the manufacturer's instructions (DynHalosperm $^{\circledR}$ Kit, Halotech DNA SL, Madrid, Spain [15]).
As per the kit instructions, sperm cells suspended in agarose were treated with an acidic solution followed by a lysing solution. Sperm cells without DNA fragmentation were observed to have long loops that formed a rich nucleic acid "halo" of decompressed DNA; those with fragmented DNA had very little or no "halo." For each sample, 300 sperm cells were counted. From these samples, the sperm DNA fragmentation index (DFI) was calculated as the percentage of cells with detectable sperm fragmentation. DNA fragmentation index in the samples was determined immediately after liquefaction ( $0 \mathrm{~h} \mathrm{DFI})$, and again after $3 \mathrm{~h}$ (3 h DFI).

\section{The rationale for the time points used}

To estimate the time point for the sperm analysis, an online questionnaire to survey professional sappers and people using metal detector hobbyists was generated. The survey was conducted in 2014, and was sent to 300 hobbyists, whose e-mails were gathered from the Internet forums, and 100 professional military engineers. Those surveyed were asked to estimate the time they usually spent using the radiating devices. Our survey results confirmed $3 \mathrm{~h}$ to be the average length of the daily exposure; hence, this time point was chosen for our second measurement.

\section{Exposure system}

After initial examination, each participant's semen sample was divided into 2 aliquots (control and experimental) and placed in a $15 \times 6 \mathrm{~mm}$ plastic tube (Eppendorf, Hamburg) in separate automated incubators with $5 \% \mathrm{CO}_{2}$ at $37^{\circ} \mathrm{C}$ (Figure 2). The samples constituting the experimental group were placed into the exposure system that emitted $43-\mathrm{kHz}$ electromagnetic waves. Sperm motility was assessed at $3 \mathrm{~h}, 12 \mathrm{~h}$ and $24 \mathrm{~h}$.

Before conducting this research, the EMFs emitted by several metal detectors had been measured. The first stage of the study concerned the identification of signal which powers the antenna. The second step was to determine the field strengths (electric and magnetic). 
For the measurement of signal parameters, a Rigol DS 1102E digital oscilloscope was used. All tested detectors used the square-wave signal with a frequency-modulated. The highest identified frequency signal was $42.74 \mathrm{kHz}$. This signal was the primary mode of operation of the detector. Therefore, it became the starting point. The exposure system (the GFG-3015 high precision programmable function generator and coil FBS 800 produced by Minelab, Cork, Patent US 4890064 A) worked at the frequency of $42.74 \mathrm{kHz}$. To determine the parameters of signal the oscilloscope was used. Parameters of the induced signal were shown in the images - screenshots of the digital oscilloscope Rigol DS 1102E (Figure 2). The identification of the measured signal (voltage) showed a rectangular shape. Small peaks in signals were related to the electromagnetic interference.

For repeatable production of magnetic field, a programmable function generator, that had characteristics equivalent to the Minelab Explorer SE generator, was used. The system was targeted to provide sufficient homogeneity in the exposure area. The electromagnetic wave was emitted

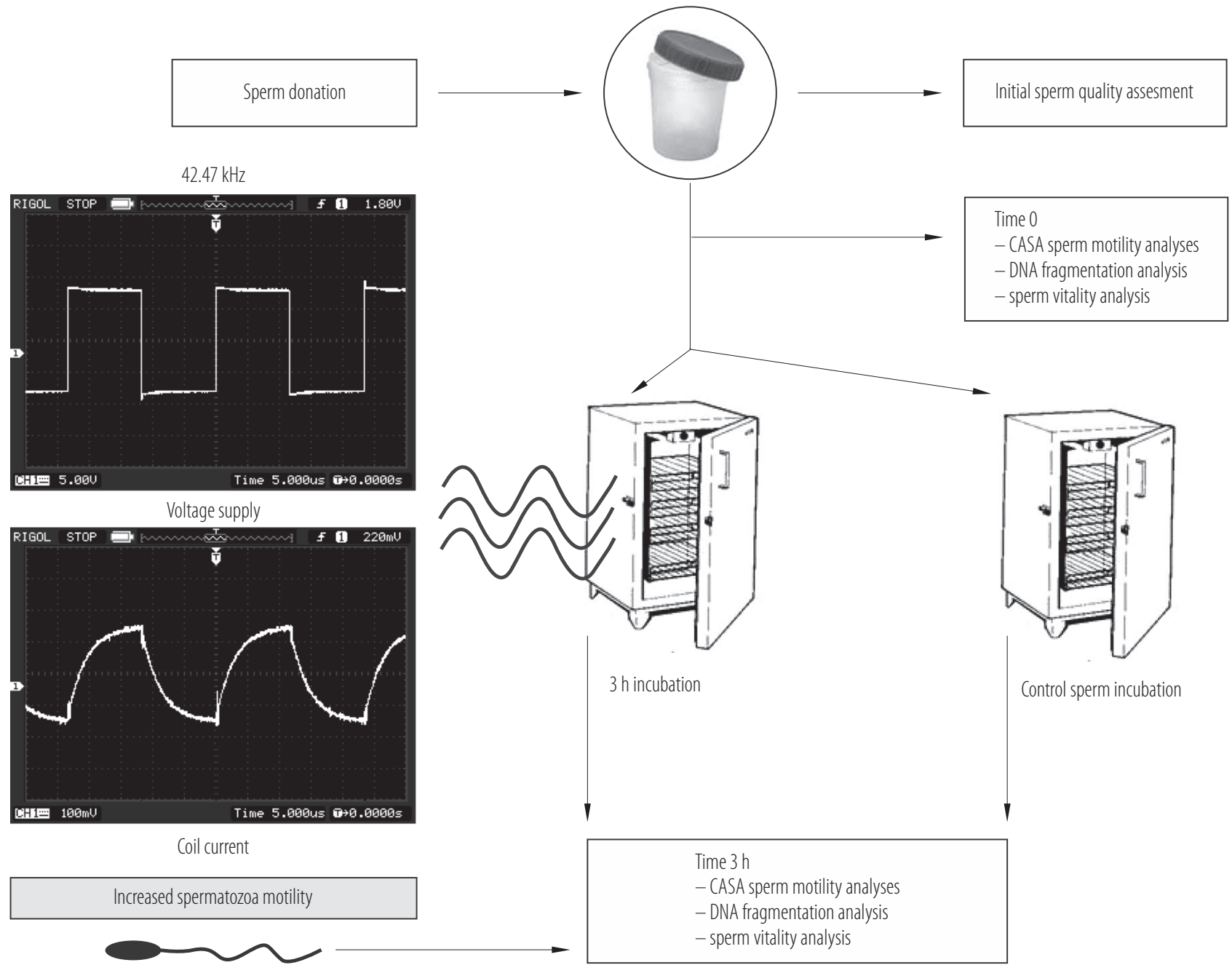

CASA - complete-aided sperm analysis.

Fig. 2. Workflow of the experiment 
by the FBS800 solenoid coil (diameter $18.5 \mathrm{~cm}$ ). Resistance $\mathrm{R}=1 \Omega$ and inductance $\mathrm{L}=0.5 \mathrm{H}$ of coil were measured by LCR-Meter ESCORT ELC 133A. The voltage of that coil signal was $8 \mathrm{~V}$ (parallel to the signal generator). In addition, the voltage measured at the resistor $(0.1 \Omega)$ connected in line with the generator. This measurement showed the shape of the current (Figure 2).

The electrical signal parameters were monitored every $2 \mathrm{~h}$. To determine the strength of the electrical and magnetic fields and their uniformity, additional measurements were performed using the ESM100 meter equipped with an isotropic sensor of EMF. That meter measures both the electrical field and the magnetic components in the range of $5 \mathrm{~Hz}-400 \mathrm{kHz}$ in 3 spatial directions (x, y, z). Accuracy of measurement is $\pm 5 \%$, and the type of values are RMS.
At a distance of $1 \mathrm{~cm}$ from the transmitting coil, the distribution of the magnetic flux density was measured. Soft ESM100 (dedicated to meter) was used for the presentation of measurement values. At a distance of at least $2 \mathrm{~m}$ from active coils, the flux density corresponded with electromagnetic background. Measurements showed some heterogeneity of the magnetic flux density. Values ranged 10-160 $\mu \mathrm{T}$ and are shown in the Figure 3.

The test tube with the sperm is several times smaller than the transmitting coil. Since the full distribution was known, it was easy to identify a smaller area of the stability of the magnetic induction. To test, an area of $50 \mathrm{~A} / \mathrm{m}$ for the magnetic field strength was chosen, which corresponded to the magnetic flux density, approx. $63 \mu \mathrm{T}$. Selecting an area was due to technical reasons of the exposure system.

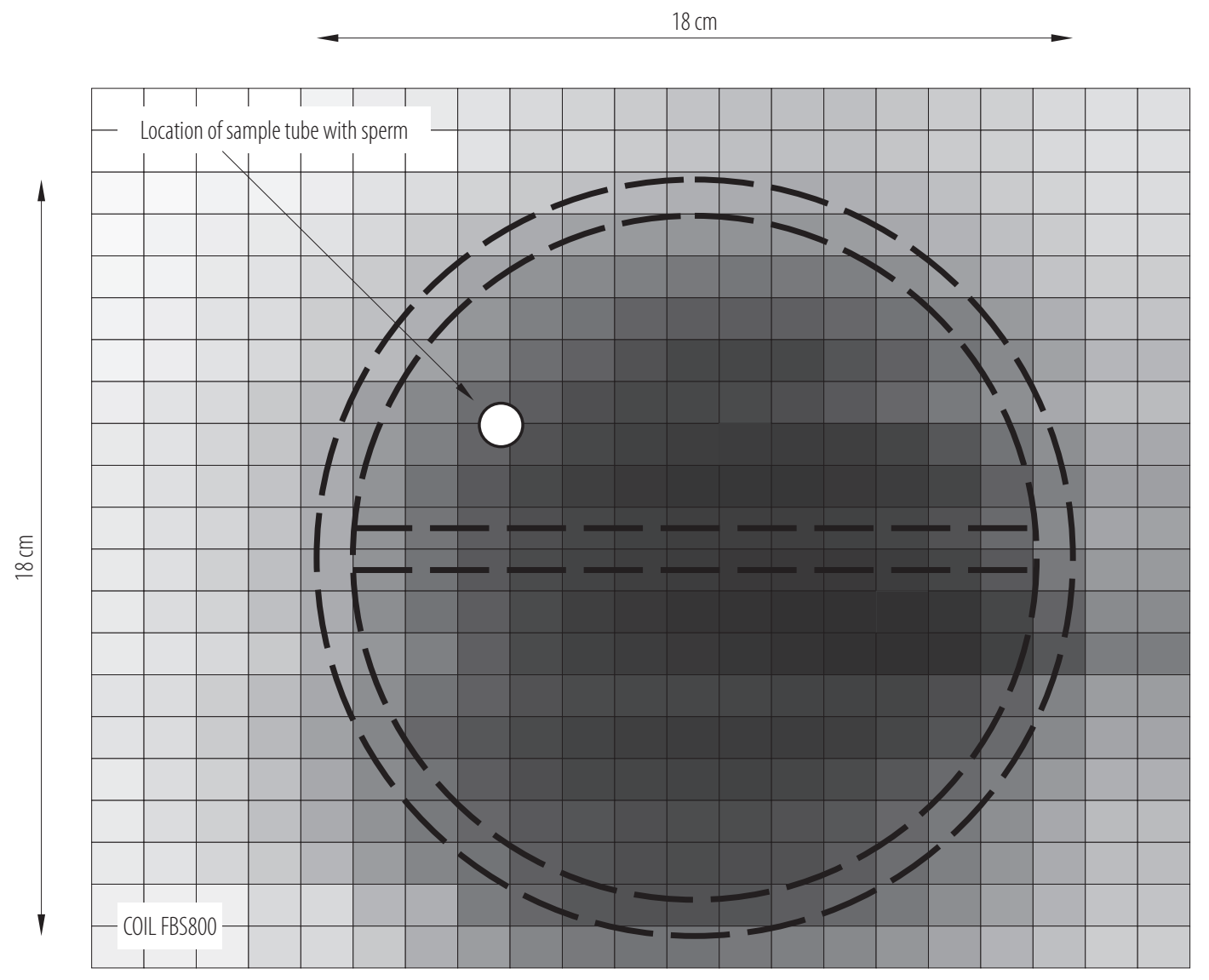

Magnetic field $[\mu T]$

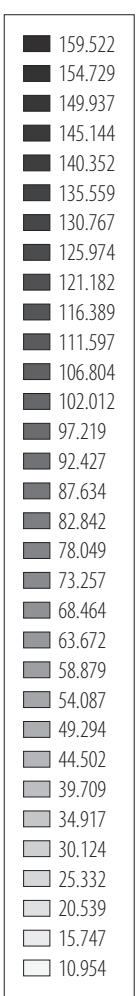

Fig. 3. Distribution of the magnetic flux density of the transmitting coil FBS800 


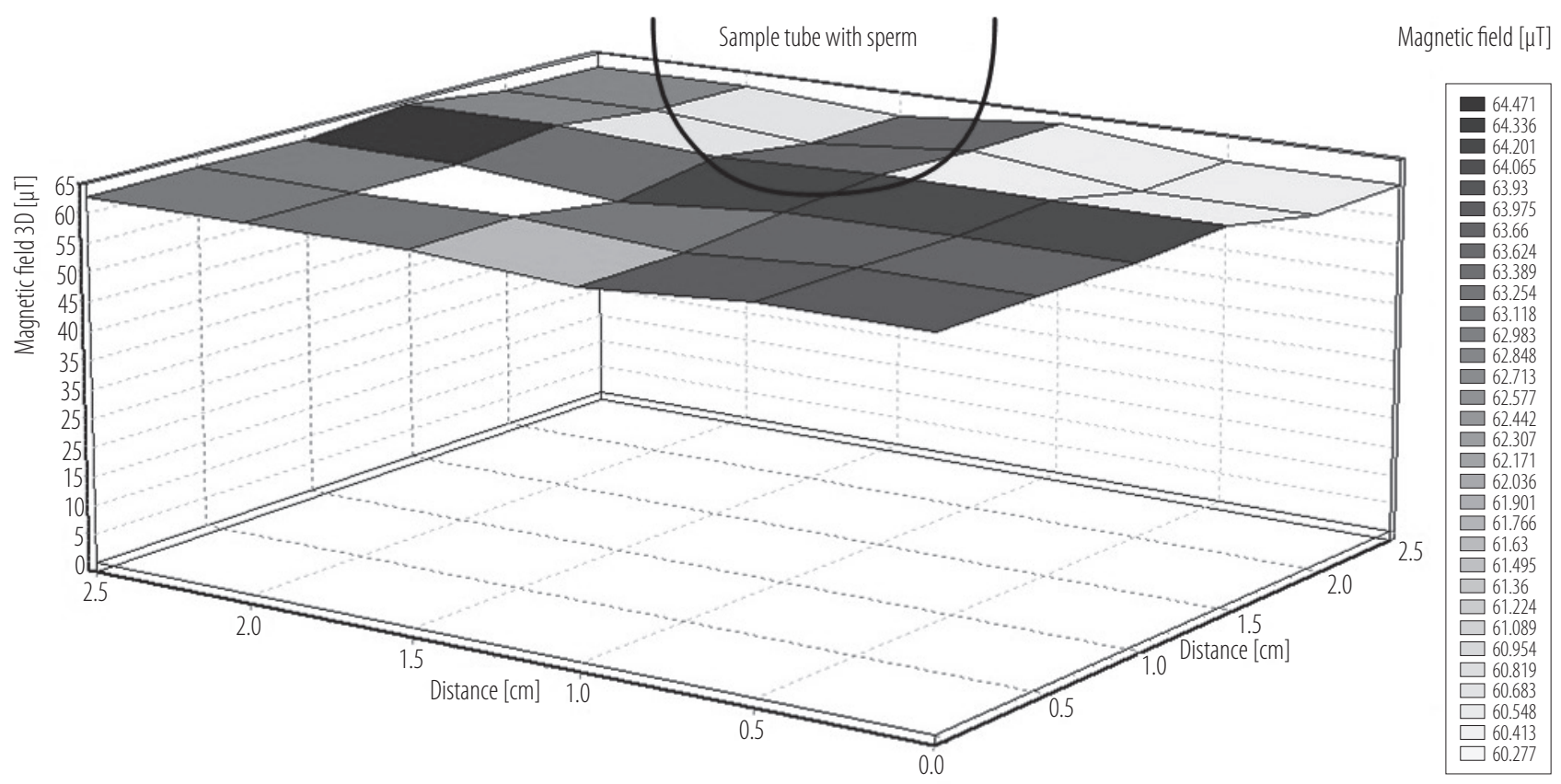

Fig. 4. Distribution of the magnetic flux density with the location of sample tube with sperm

Not every place allowed to put the tube. The location of the tube with sperm is marked in the Figure 3. The Figure 4 shows the distribution of the magnetic flux density in the nearest space. Tolerance of magnetic flux density value is in the range of $\pm 5 \%(2.33-4.32 \%)$.

\section{Sperm vitality test}

Sperm vitality was assessed using eosin staining and the hypo-osmotic swelling test, according to protocols described in the WHO laboratory manual for the analysis of human semen [14].

\section{Statistical methods}

The data was statistically analyzed using Statistica 9.1 software (StatSoft, Poland). Mean values (M) with standard deviations (SD), median values with the (min.-max) range, lower and upper quartiles were estimated and presented in the Table 1 whereas mean values with standard deviations were presented in the Figures 5-8. Since the treated and tested groups were made of the same samples divided prior
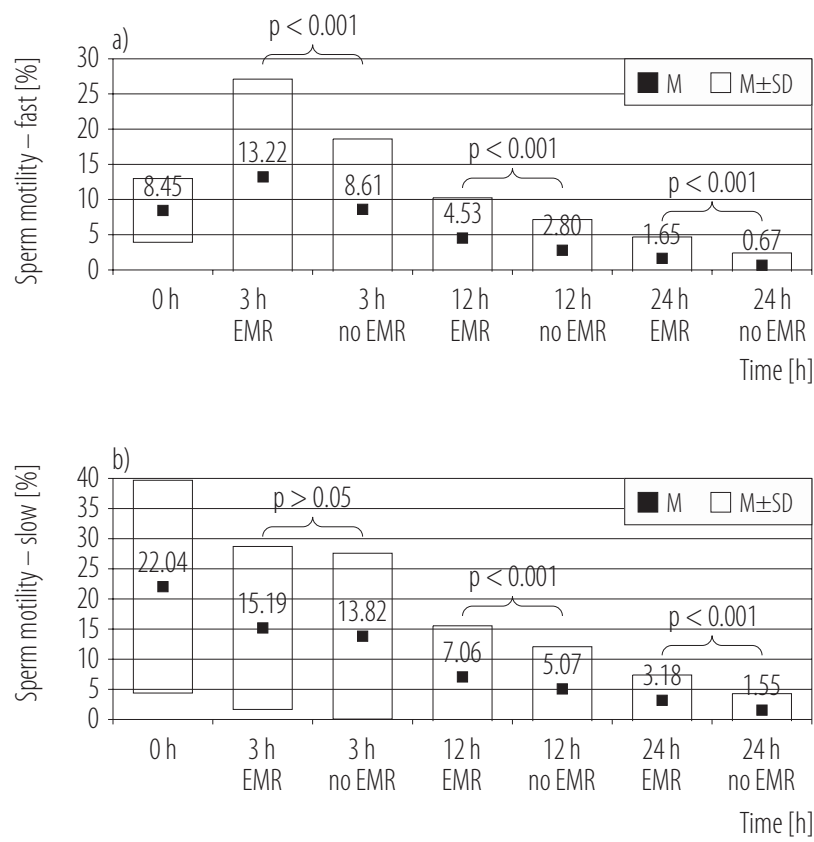

M - mean; SD - standard deviation.

Fig. 5. Comparison of the percentage share of sperm displaying a) fast and b) slow progressive motility at different time points after sperm donation with and without electromagnetic radiation (EMR) 


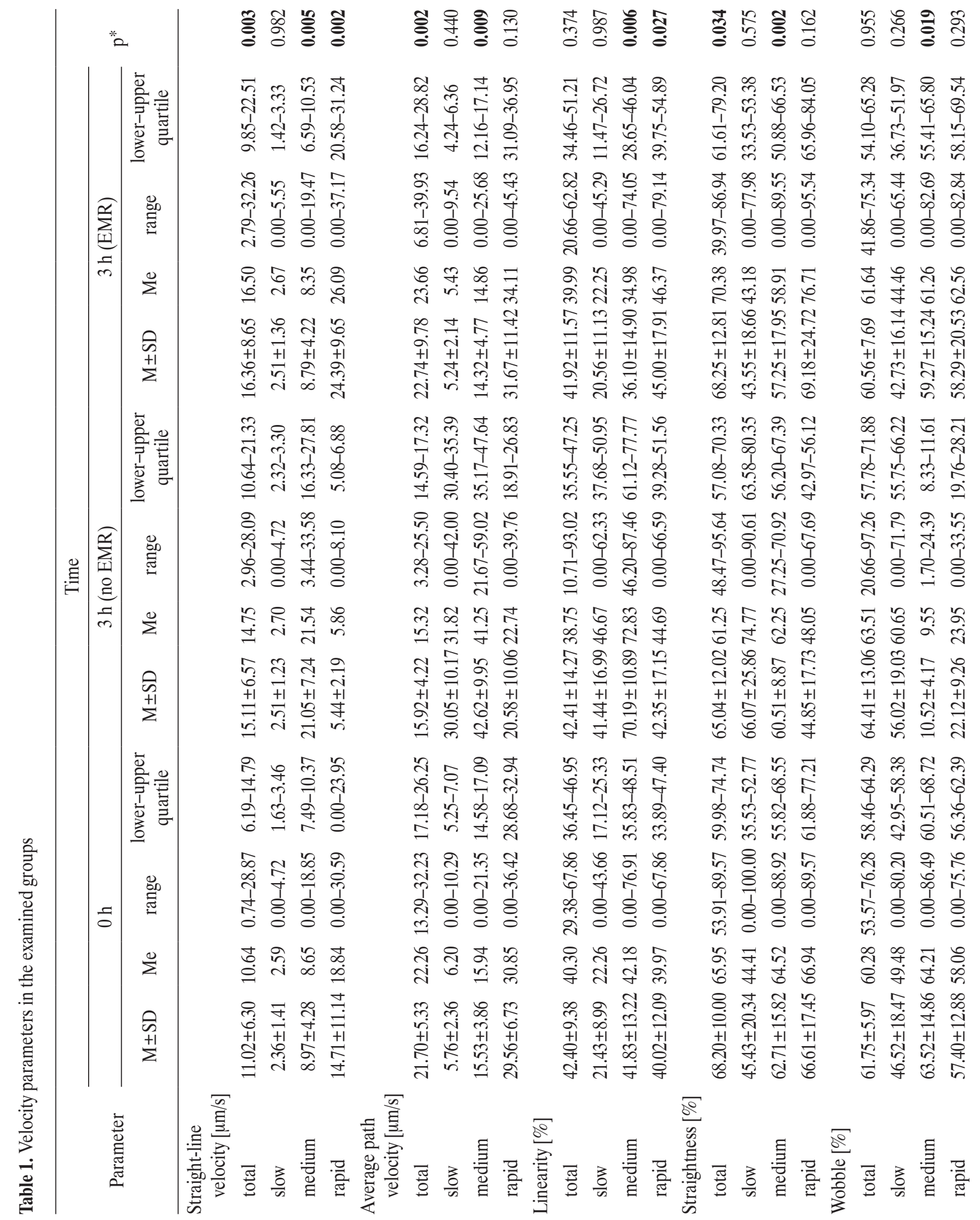




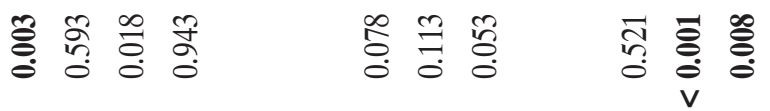

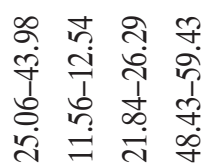

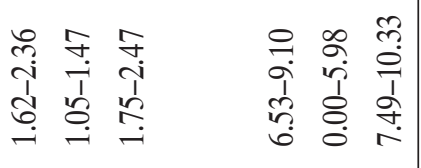

๖. ชู กิ สุ

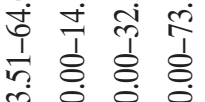

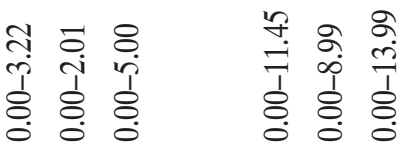

끄 증

๓ं ฮ่

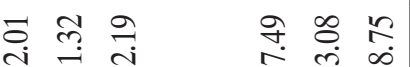

กิ กิ ซุ

$+1+1+1$

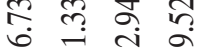

尺

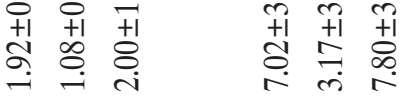

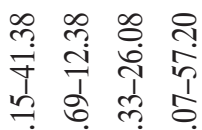

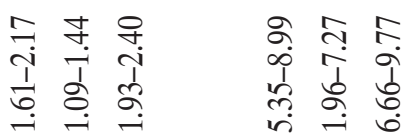

๙

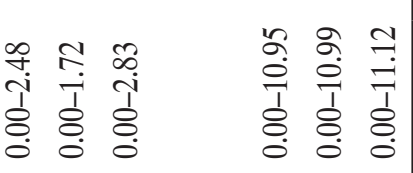

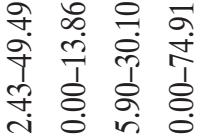

๙ૅ

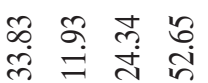

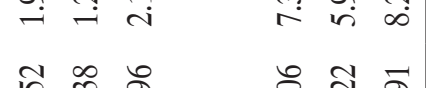

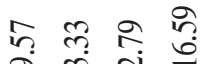

$+1+1+1$

少学

लं

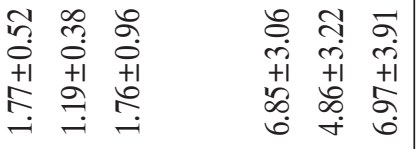

$\infty$ ำำ

ๆ

ป⿻

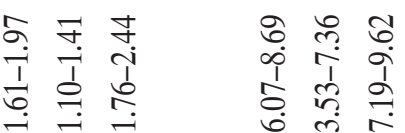

กิ ปี

क ম

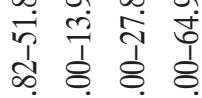

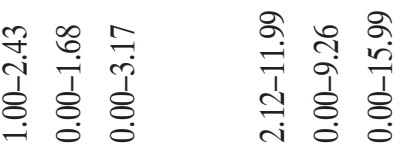

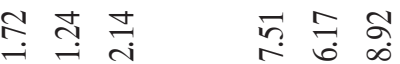

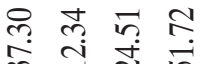

๗ $\overrightarrow{0}$

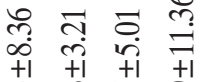

กิ

थं

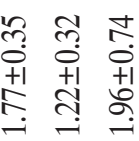

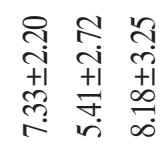

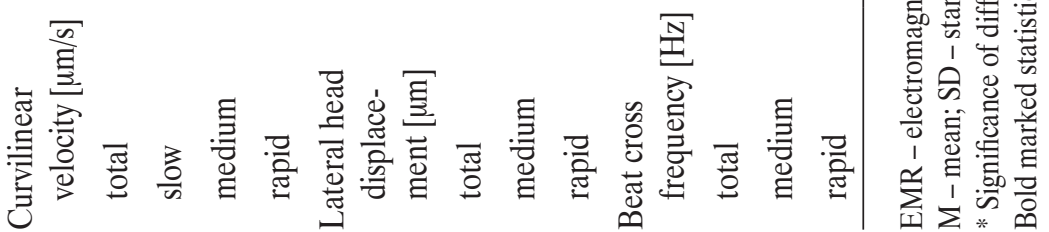




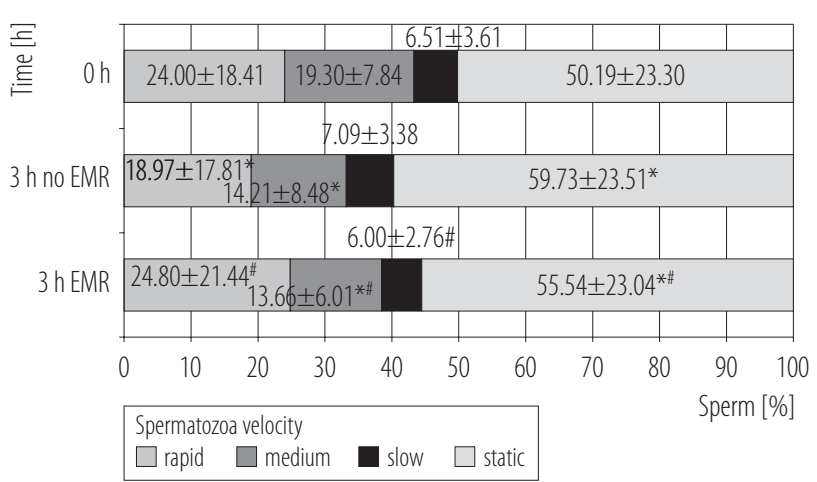

* Significantly different from $0 \mathrm{~h}$.

\# Significantly different from $3 \mathrm{~h}$ with no EMR.

Abbreviations as in Figure 5.

Fig. 6. Comparison of the percentage share of sperm displaying slow progressive motility at different time points after sperm donation with and without electromagnetic radiation (EMR)

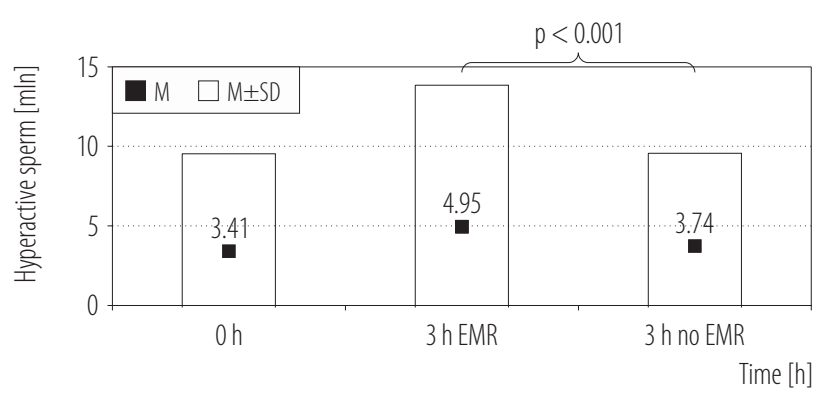

* Significantly different from $0 \mathrm{~h}$.

\# Significantly different from $3 \mathrm{~h}$ with no EMR.

Fig. 7. Comparison of the percentage share of sperm attributed to different velocity groups (rapid, medium, slow and static) in the $3 \mathrm{rd} \mathrm{h}$ after sperm donation with and without electromagnetic radiation (EMR)

to the experiment, t-test for paired samples was used. The parameter estimators were asymptotically normal distributed due to the central limit theorem for large sample size. The $\mathrm{p}<0.05$ value was considered as a significant difference.

\section{RESULTS}

\section{Quality of the sperm samples}

Before performing sperm sample aliquoting (to establish experimental and control groups), the sample quality analysis was carried out. The average concentration of sperm

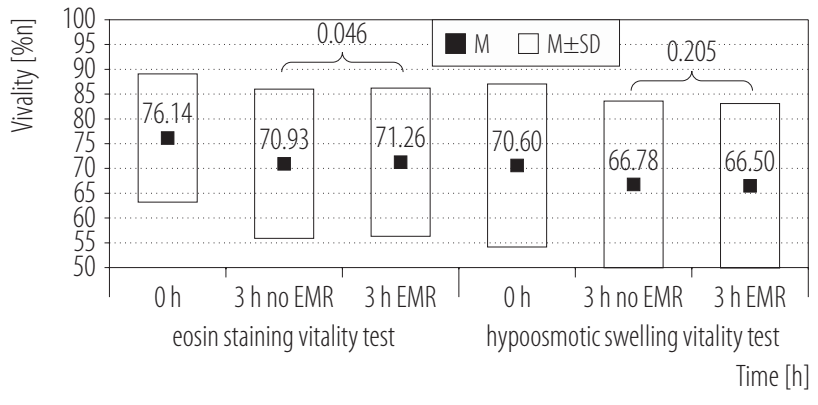

Abbreviations as in Figure 5.

Fig. 8. The vitality of spermatozoa at the beginning of the experiment and after $3 \mathrm{~h}$ of incubation with or without electromagnetic field

was found to be $40.49 \pm 18.87 \mathrm{mln} / \mathrm{ml}$, and the percentage share of spermatozoa without morphological defects was $8.74 \pm 4.49 \%$. Out of the 300 spermatozoa assessed (per sample), $81.09 \pm 9.73 \%$ passed the HBA. These results enabled us to conclude that our samples were of satisfactory quality, according to the WHO reference values [14].

\section{LF-EMR increases the percentage of sperm in rapid progressive motility}

During the assessment of sperm motility, it was noted that exposure to EMR caused an increase in the percentage of spermatozoa displaying fast progressive motility. This difference was mostly visible during the first hours of incubation, during which time percentage share of "type a" spermatozoa increased from around $10 \%$ to more than $13 \%$ in the irradiated group. After $3 \mathrm{~h}$, this led to a significant difference in the percentage of "type a" spermatozoa between the irradiated and control groups $\left(13.22 \%\right.$ vs. $\left.8.61 \% ; \mathrm{t}=-7.458 ; \mathrm{p}<10^{-4}\right)$ (Figure $\left.5 \mathrm{a}\right)$. Over the next $21 \mathrm{~h}$ (until the 24-h time point), this difference gradually decreased, but remained statistically significant (12 h - experimental: $4.52 \%$ vs. control: $2.8 \%$; $\mathrm{t}=-5.888$; $\mathrm{p}<10^{-4} ; 24 \mathrm{~h}$ - experimental: $1.65 \%$ vs. control: $0.67 \%$; $\left.\mathrm{t}=-6.195 ; \mathrm{p}<10^{-4}\right)$. Although no statistically significant difference in the percentage share of "type b" spermatozoa was observed between the irradiated and control groups 
after $3 \mathrm{~h}$ of incubation (Figure 5b), at $12 \mathrm{~h}$ and $24 \mathrm{~h}$ this difference reached significance $(12 \mathrm{~h}$ - experimental: $7.06 \%$ vs. control: $5.07 \% ; \mathrm{t}=-4.315 ; \mathrm{p}<0.005 ; 24 \mathrm{~h}-$ experimental: $3.18 \%$ vs. control: $1.55 \%$; $\left.\mathrm{t}=-6.616 ; \mathrm{p}<10^{-4}\right)$.

\section{LF-EMR slows the decrease in rapid sperm velocity during first $3 \mathrm{~h}$ of incubation}

In order to estimate the impact of EMR on spermatozoa velocity, the samples at time 0 and after $3 \mathrm{~h}$ of irradiation or incubation in the control environment were analyzed. Based on their velocity, the spermatozoa were divided into 4 groups: rapid, medium, slow and static. The 3-h incubation led to a decrease in the percentage of rapid- and medium-velocity spermatozoa. Initially, $19.3 \%$ of sperm was assigned to the medium-velocity group; however, this value decreased significantly $(\mathrm{p}<0.001)$ in both groups after the 3 -h incubation, reaching $14.21 \%$ in the control and $13.66 \%$ in the group exposed to the EMF. Surprisingly, the percentage share of rapid-velocity spermatozoa remained unchanged after $3 \mathrm{~h}(0 \mathrm{~h}-24 \%$ vs. $3 \mathrm{~h}$ - experimental: $24.8 \%$; control: $18.97 \% ; p=$ not statistically significant) (Figure 6). The average percentage share of slow spermatozoa was $6.51 \%$ at time 0 , and this did not change significantly after $3 \mathrm{~h}$ of incubation (experimental: $6 \%$ vs. control: 7.09\%). Interestingly, however, the difference in the percentage of slow-velocity spermatozoa between the groups was statistically significant at this time point $(\mathrm{p}=0.002)$. After the 3 -h incubation, the average percentage of static sperm increased significantly in both groups ( 0 h: $50.19 \%$ vs. 3 h control: $59.73 \%$; $p<0.001$ or vs. 3 h experimental: $59.73 \%$; $=0.002$ ). A higher average percentage of static sperm was observed in the control samples $(59.73 \%)$ compared with those treated with electromagnetic waves (55.54\%), with this difference being statistically significant $(\mathrm{p}<0.001)$.

\section{LF-EMF affects sperm velocity parameters}

All velocity analyses were performed at the beginning of the experiment $(0 \mathrm{~h})$ and after $3 \mathrm{~h}$ of incubation, both with and without the presence of the EMF. The average value of the VSL total after $3 \mathrm{~h}$ of incubation was significantly higher in the irradiated group compared to the control group $(16.36 \mu \mathrm{m} / \mathrm{s}$ vs. $15.53 \mu \mathrm{m} / \mathrm{s}$, respectively; $\mathrm{t}=-3.07$; $\mathrm{p}=0.003$ ). The VSL slow values reached $2.51 \mu \mathrm{m} / \mathrm{s}$ in both groups. The VSL rapid value was significantly higher in the group exposed to the EMF compared to the control group $(24.39 \mu \mathrm{m} / \mathrm{s}$ vs. $22.12 \mu \mathrm{m} / \mathrm{s}$, respectively; $\mathrm{t}=2.857$; $\mathrm{p}=0.005)$. Straight-line velocity medium values, on the other hand, were higher in the control group than the irradiated group $(10.52 \mu \mathrm{m} / \mathrm{s}$ vs. $8.79 \mu \mathrm{m} / \mathrm{s}$, respectively; $\mathrm{t}=2.857 ; \mathrm{p}=0.005)$.

Similarly to the VSL total, after 3 h of incubation, the VCL total value was significantly higher in the irradiated group than the control group $(36.73 \mu \mathrm{m} / \mathrm{s}$ vs. $33.97 \mu \mathrm{m} / \mathrm{s}$, respectively; $\mathrm{t}=-3.068 ; \mathrm{p}=0.002)$. The opposite was true for VCL medium: a significantly higher average value was recorded in the control group than the irradiated group $(24.35 \mu \mathrm{m} / \mathrm{s}$ vs. $22.94 \mu \mathrm{m} / \mathrm{s}$, respectively; $\mathrm{t}=2.406$; $\mathrm{p}=0.593)$. The analysis of VCL slow and VCL rapid values for irradiated and control sperm samples revealed no statistically significant differences.

The VAP total value was higher in the group treated with EMR than the control group $(22.74 \mu \mathrm{m} / \mathrm{s}$ vs. $21.05 \mu \mathrm{m} / \mathrm{s}$, respectively; $\mathrm{t}=-3.138 ; \mathrm{p}=0.002)$. Analogous to the relationship between VCL total and VCL medium, the VAP medium value was higher in the control group than the irradiated group $(15.92 \mu \mathrm{m} / \mathrm{s}$ vs. $14.32 \mu \mathrm{m} / \mathrm{s}$, respectively; $\mathrm{t}=2.659 ; \mathrm{p}=0.002)$. No statistically significant differences in the VAP slow and VAP rapid values were observed between the groups.

The LIN rapid coefficient was higher for samples exposed to EM waves than those without (44\% vs. $41.44 \%$, respectively; $\mathrm{t}=2.793 ; \mathrm{p}=0.006)$. Conversely, the LIN medium value was higher in the control group than the irradiated group ( $42.41 \%$ vs. $36.10 \%$, respectively; $t=2.793$; $\mathrm{p}=0.006)$. The LIN total and LIN average values did not differ significantly between the groups. 
The average STR coefficients in the control group reached $70.19 \%$ for the STR total and $65.04 \%$ for the STR medium, both of which were higher than those observed in the irradiated groups $(68.24 \%$ and $57.25 \%$, respectively). No statistically significant differences for STR slow and STR rapid were observed between groups.

The ratio of WOB total, WOB rapid and WOB slow did not differ significantly between the 2 groups. Wobble medium was higher in the control group (64.41\%) compared to the EMR-exposed group (59.27\%), and this difference was statistically significant $(\mathrm{t}=2.385 ; \mathrm{p}=0.019)$.

\section{LF-EMR affects sperm beat cross frequency but has no effect on lateral head displacement}

In the case of ALH total, ALH medium progressive and ALH rapid progressive, no statistically significant differences between the 2 groups were observed. Among ejaculates exposed to the EMF, the BCF rapid progressive coefficient was $7.8 \mathrm{~Hz}$, as compared to $6.97 \mathrm{~Hz}$ in the control group; the difference between these values was statistically significant $(t=-2.705 ; p=0.008)$. The inverse relationship was observed for BCF medium progressive, where the value of the control group reached $4.86 \mathrm{~Hz}$, as compared to $3.17 \mathrm{~Hz}$ in the study group. Again, this difference was statistically significant $(t=4.646 ; p<0.005)$. Beat cross frequency total did not differ significantly between the 2 groups (Table 1 ).

In the ejaculates studied, at $0 \mathrm{~h}$ the average total quantity of sperm with hyperactivation features amounted to 3.412 million. After $3 \mathrm{~h}$ of exposure to the EMF, the number of hyperactive spermatozoa increased to 4.951 million whereas in the control group it increased to only 3.735 million. The difference between these values was statistically significant $(\mathrm{t}=-3.09 ; \mathrm{p}=0.003)$ (Figure 7).

\section{LF-EMR did not affect spermatozoa DNA fragmentation}

To check for possible effects of EMR on chromatin fragmentation, a chromatin dispersion test was used. At the beginning of the experiment, the DFI was recorded as $14.58 \pm 8.18 \%$. After $3 \mathrm{~h}$ of incubation, this value significantly increased in both the control $(42.32 \pm 5.73 \%)$ and irradiated $(41.77 \pm 6.07 \%)$ groups; however, this difference was not statistically significant. No statistically significant relationship between exposure to EM and chromatin fragmentation in spermatozoa was found.

\section{LF-EMR may affect sperm vitality}

In order to estimate cell vitality and membrane integrity, 2 reference vitality tests (eosin staining and the hypoosmotic swelling test) were conducted, as described in the WHO manual [14]. Initial (0 h) sperm vitality, according to the results of hypo-osmotic swelling test, was assessed as $70.6 \%$. After $3 \mathrm{~h}$, vitality decreased to $66.78 \%$ in the control group and $66.5 \%$ in the irradiated group. Statistical assessment of the results of this test did not provide evidence to support the existence of any convincing causeeffect relationship between EMR and sperm viability. Nevertheless, statistically significant differences were observed using the eosin staining method. At the beginning of the experiment $(0 \mathrm{~h})$ cell viability, as assessed by dye inclusion, was $76.14 \%$; after $3 \mathrm{~h}$ this value decreased to $71.26 \%$ in the irradiated group and $70.93 \%$ in the control group. The difference between these values was found to be statistically significant $(t=2.02 ; p=0.046)$ (Figure 8$)$.

\section{DISCUSSION}

It has been shown that exposure to $43-\mathrm{kHz}$ electromagnetic waves increases the percentage share of sperm displaying progressive motility, establishing a clear relationship between LF radiation and sperm motility for the first time. Studies published to date focused on the impact of waves with lower $(10 \mathrm{~Hz}, 25 \mathrm{~Hz}, 50 \mathrm{~Hz})$ or higher frequencies (> $800 \mathrm{MHz}$ ). Iorio et al. [16,17], Roychoudhury et al. [18], Falahati et al. [19], Łopucki et al. [20], and Formicki et al. [21] found evidence to support the relationship between EMR and increased sperm motility. Falahati et al. found that exposing human semen samples to a frequency 
of $10 \mathrm{~Hz}$ for $4 \mathrm{~h}$ led to a 1.8-fold increase in quick-motile sperm [19]. The same study revealed that the percentage share of slow-motile sperm decreased by $40 \%$ after $2 \mathrm{~h}$. The authors obtained similar results for a 4-h $25-\mathrm{Hz}$ irradiation, where quick motility was observed to increase 1.6-fold. The same study also confirmed $2 \mathrm{~h}$ of $10-\mathrm{Hz}$ irradiation to have a small but significant impact on sperm vitality [19]. Lopucki et al. found a higher percentage share of sperm displaying fast motility, and a decrease in those displaying slow motility, in human ejaculates exposed to an EMF of $50 \mathrm{~Hz}, 0.5 \mathrm{mT}$ for $2 \mathrm{~h}$ [20]. Studies by Roychoudhury et al. [18], using rabbits, confirmed the beneficial effects of exposure to $50-\mathrm{Hz}$ radiation on sperm motility. The authors found that when exposed to EMR, the VCL value of the sperm increased and, in their case, so did the number of fertilizations after insemination using semen exposed to EMR, too [18]. This is consistent with a study by Formicki et al., who observed an improvement in sperm motility and an increased percentage share of fertilizations after exposing fish reproductive cells to magnetic fields of $1.5 \mathrm{mT}$ and $10 \mathrm{mT}$ for $24 \mathrm{~h}$ [21]. Their study, similarly to ours and that of Roychoudhury et al. [18], was conducted using the CASA system and showed EMR exposure to have a positive effect on sperm motility. A similar relationship was observed for VSL, VAP and ALH parameters, which were gradually elevated in response to increasing field intensity. In the case of LIN and STR, the increase in field intensity resulted in an initial increase followed by a decrease in the average values of these parameters [18]. Iorio et al. showed that significant increases in motility values and other kinematic parameters had been observed when spermatozoa had been exposed to an ELFEMF with a square waveform of $5 \mathrm{mT}$ amplitude and a frequency of $50 \mathrm{~Hz}$ [16]. The results obtained by Iorio et al. were consistent with our observations but our research involved a different characteristic of the EMF. In their subsequent research, Iorio's team demonstrated that sperm exposure to ELF-EMF resulted in a progres- sive and significant increase of mitochondrial membrane potential and levels of adenosine triphosphate (ATP), adenosine diphosphate (ADP) and nicotinamide adenine dinucleotide $\left(\mathrm{NAD}^{+}\right)$that were associated with a progressive and significant increase in the sperm kinematic parameters [17].

Contrasting conclusions were drawn by $\mathrm{Xu}$ et al. who studied semen after exposure to a 50-Hz EMF at $0.4 \mathrm{mT}$ and found that 15 - and $60-\mathrm{min}$ exposures resulted in a reduction in the percentage share of sperm displaying fast progressive motility [22]. Similar results were obtained by Bernabò et al. who found that in vitro exposure of boar spermatozoa to a 50-Hz extremely low frequency (ELF)EMF of $>0.5 \mathrm{mT}$ induced progressive acrosome damage, which compromised the ability of spermatozoa to undergo acrosomal reaction after zona pellucida stimulation, and reduced the success rate of in vitro fertilization [23]. These effects became evident at $0.75 \mathrm{mT}$ and plateaued at $1 \mathrm{mT}$. Under in vivo conditions, an ELF-EMF intensity of $1 \mathrm{mT}$ was found to compromise sperm function, significantly reducing the fertilization rate [23]. In an earlier study Bernabò et al. found ELF to have a negative influence on spermatozoa, mostly due to impaired cell calcium homeostasis [24].

Mobile phones emit EMR at frequencies between $800 \mathrm{MHz}$ and $2200 \mathrm{MHz}$, which may be absorbed by the human body. Earlier studies showed that prolonged in vivo exposure to electromagnetic waves associated with mobile telephony lowers sperm motility [25], which was in line with studies reviewed by Adams et al [26]. In their article, Adams et al. analyzed 9 in vivo and in vitro studies, which altogether included 1448 samples collected from 1353 men. Six out of these studies showed a significant negative effect of mobile phone exposure on human sperm motility. Liu et al. performed a similar analysis of 18 studies conducted on a total of 3947 men and 186 rats [27]. Twelve of the studies in their analysis (4 human, 4 in vitro, and 4 animal studies) were conducted on 1533 men and 97 rats and used for the detailed 
meta-analysis. Overall, this systematic review showed that the majority of human and in vitro laboratory studies indicated mobile phone use or RF exposure to have a negative effect on semen. The meta-analysis of the human studies alone indicated that mobile phone usage had no adverse effects on semen parameters; whereas, in the in vitro studies, RF radiation had a detrimental effect on sperm motility and vitality. Similarly, the animal studies revealed that RF exposure had harmful effects on sperm concentration and motility. The results of epidemiological studies on exposure to EMF and semen quality present different results and undoubtedly, this issue will require further research in the future, which could be carried out using individual dosimeters [11].

Wi-Fi operates at frequencies $2400-2485 \mathrm{MHz}$ and $4915-$ $5825 \mathrm{MHz}$. Studies published by Avendaño et al. [28], Freour et al. [29], and Yildirim et al. [30] demonstrate the negative impact of frequencies in this range on sperm motility. The defects in sperm motility were observed as well after exposing testicular tissues to irradiation as after in vitro irradiation of semen.

The 43-kHz electromagnetic wave used in our study belongs to the LF spectrum, which has not been yet thoroughly tested for its impact on sperm. According to previously published literature, at frequencies higher and lower than the one that has been used, the harmful effects of electromagnetic waves tend to be more pronounced. This may be explained by the fact that a living cell is a source of a natural electrical field, with a certain capacity and resistance. In view of the biophysical properties of cell membranes, one can assume that its capacitance begins to be omitted by waves in the range of $10 \mathrm{kHz}-$ $100 \mathrm{MHz}$; therefore, LF-EMR is not transferred into the cell and affects only the cell membrane [31]. The EMF of high frequencies, on the other hand, will primarily affect the internal structure of the cell. Electromagnetic waves may affect the balance in the oxidoreductive system and the functioning of ion channels, which are jointly responsible for the process of sperm hyperactivation. A shift in the balance of the oxidoreductive system is necessary to trigger the capacitation.
The presence of reactive oxygen species (ROS), which subsequently react with capacitation regulatory proteins, is necessary to start the process of capacitation but an overabundance of ROS could cause premature capacitation or even damage to the sperm DNA and enzymes [32].

Proper functioning of ion transport across the cell membrane is critical for the maturation of sperm in the reproductive tract, and affects the ability of the sperm to fertilize the ovum. The following ion channels are involved in sperm maturation: CatSper, pH-regulated, calcium-selective ion channel, KSper (Slo3) and the HV1 voltage-gated channel [33]. The HV1 voltage-gated channel is believed to be the most vulnerable to electromagnetic waves. This channel is responsible for the alkalization of the intracellular environment and is necessary for capacitation, acrosome reaction, hyperactivation and sperm motility. Walleczek [34] has found that EMR is able to modify the transport of calcium ions into the cells of the immune system, and a wave frequency of $\sim 15 \mathrm{~Hz}$ has the greatest influence on this phenomenon. It may therefore be expected that, similarly to irradiated immune system cells, ion transport impairment may be also present in the irradiated sperm, which may partially explain our findings of improved sperm motility parameters in the EMR-treated sperm [34]. Stimulation of the HV1 voltage-gated channel but also oxidative stress and their related events appear to be a potential mechanism involved in increased sperm motility. However, the detailed mechanisms of this phenomenon remain unknown. This will require further research in the future. Apart from our observation of increased motility of sperm following irradiation, beneficial effects of LF-EMR exposure have been reported by others in various biological systems. Lim et al. found that $10-\mathrm{Hz}$ and $50-\mathrm{Hz}$ radiation promoted the growth of stem cells derived from pig [35]. A similar phenomenon was observed by Liu et al. in mesenchymal stem cells derived from rat bone marrow: proliferation of these cells was significantly increased in the group exposed to waves of $10 \mathrm{~Hz}$ [36]. Interestingly, in 2010, a method of 
treatment using EMF-stimulated mesenchymal stem cells was patented in the USA (Patent No. 7744,869 B2).

Our results of improved sperm motility after $3 \mathrm{~h}$ of exposure to electromagnetic waves are of great significance because this time period was declared as the average daily exposure experienced by professional sappers and those whose hobby was metal detecting. Furthermore, as shown by Wang et al., hyperactivated motility reaches its peak at $1 \mathrm{~h}$ and flattens after $3 \mathrm{~h}$ of incubation [37]. Therefore, it could be postulated that low intensity LF irradiation may serve as a part of a novel medical protocol that would enable the rapid motility of spermatozoa to be sustained during assisted reproductive technology procedures.

Previous studies have shown that sperm VCL values assessed using CASA are highly correlated with the efficacy of in vitro fertilization techniques in humans [38]. A positive correlation has also been shown to exist between VCL, VSL and the number of successful fertilizations in animal models [39]. Our research therefore suggests that LF-EMR could increase the ability of sperm to fertilize an egg cell in vivo. Despite this possibility, drawing concrete conclusions as to whether this effect is positive or negative in view of infertility treatment is premature. As our study was conducted in vitro, the influence of the LF-EMR on the fertility of humans exposed to LF-EMR-emitting devices on a daily basis remains an unanswered question. Nevertheless, it would be of social interest to inform the users about the possible health effects of LF-EMR overexposure. The idea of using LF-EMR to support the procedures of assisted reproductive technology or other kinds of infertility treatment also needs further detailed evaluation.

Premature capacitation is known to occur in the seminal plasma, causing energy depletion of the sperm and decreasing the chance of fertilization; therefore, increasing the severity of capacitation within an appropriate timeframe should be beneficial for male fertility. Thus far, the affect of LF-EMR on the integrity of genetic material and whether it may increase the risk of epigenetic chromatin modi- fications is also unclear [40]. In our study, no alteration in the DFI after sperm irradiation was found; therefore, our results indicate that LF-EMR does not lead to doublestrand DNA breaks or large chromatin rearrangements. Further research is needed to confirm whether other types of DNA damage, such as base oxidation, depurination of depyrimidation, are caused by LF-EMR.

\section{CONCLUSIONS}

In vivo environmental exposure to $43-\mathrm{kHz}$ waves may promote the development of infertility related to premature capacitation outside of the vaginal tract.

It has been shown that $43 \mathrm{kHz}$ is not indifferent to the processes involved in reproduction. Hopefully, data will encourage other scientists to conduct more detailed research on the biological effects of electromagnetic waves and their impact on the reproductive system.

\section{ACKNOWLEDGMENTS}

Special thanks to Krzysztof Plewka and Patrycja Sitarska for performing the CASA analysis. The authors acknowledge the assistance of Proper Medical Writing Sp. z o.o. in the proofreading and copy editing of this manuscript.

\section{REFERENCES}

1. Suarez SS, Ho HC. Hyperactivation of mammalian sperm. Cell Mol Biol. 2003;49(3):351-6.

2. Bakalczuk G, Wdowiak A, Lewicka M, Sulima M, Perovic S, Juric A, et al. Induction effectiveness of acrosome reaction in prepared human spermatozoa assessed using the CD46 surface antigen. Eur J Med Technol. 2016;1(10):44-53.

3. Carpenter DO. Human disease resulting from exposure to electromagnetic fields. Rev Environ Health. 2013;28(4):15972, https://doi.org/10.1515/reveh-2013-0016.

4. Wdowiak A, Mazurek PA, Wdowiak A, Bojar I. Effect of electromagnetic waves on human reproduction. Ann Agric Environ Med. 2017;24(1):13-8, https://doi.org/10.5604/ 12321966.1228394. 
5. Challis LJ. Mechanisms for interaction between RF fields and biological tissue. Bioelectromagnetics. 2005;Suppl 7: S98-106, https://doi.org/10.1002/bem.20119.

6. Aitken RJ, Smith TB, Jobling MS, Baker MA, De Iuliis GN. Oxidative stress and male reproductive health. Asian J Androl. 2014;16(1):31-8, https://doi.org/10.4103/1008-682X.122203.

7. Kotnik T, Miklavcic D. Theoretical evaluation of voltage inducement on internal membranes of biological cells exposed to electric fields. Biophys J. 2006;90(2):480-91, https://doi. org/10.1529/biophysj.105.070771.

8. Kotnik T, Pucihar G, Miklavcic D. Induced transmembrane voltage and its correlation with electroporation-mediated molecular transport. J Membr Biol. 2010;236(1):3-13, https://doi.org/10.1007/s00232-010-9279-9.

9. Cotgreave IA. Biological stress responses to radio frequency electromagnetic radiation: Are mobile phones really so (heat) shocking? Arch Biochem Biophys. 2005;435(1):22740, https://doi.org/10.1016/j.abb.2004.12.004.

10. Mancinelli F, Caraglia M, Abbruzzese A, d'Ambrosio G, Massa R, Bismuto E. Non-thermal effects of electromagnetic fields at mobile phone frequency on the refolding of an intracellular protein: Myoglobin. J Cell Biochem. 2004;93(1):188-96, https://doi.org/10.1002/jcb.20164.

11. Mazurek PA, Boś B, Wdowiak A, Naumchuk OM. Dosimetry of the electromagnetic fields of the GSM/UMTS/WLAN band. Eur J Med Technol. 2016;1(10):15-24.

12. Mazurek PA, Wdowiak A. [Metal detectors - Characterisation of electromagnetic emission levels]. Przegl Elektrotech. 2015;91(12):163-6, https://doi.org/10.15199/48.2015.12.41. Polish.

13. Mazurek PA, Wdowiak A, Anusiewicz A. Electromagnetic interactions of metal detectors. Eur J Med Technol. 2017;1(14):33-40.

14. World Health Organization. WHO laboratory manual for the examination and processing of human semen. 5th ed. Geneva: The Organization; 2010.

15. Fernandez JL, Muriel L, Goyanes V, Segrelles E, Gosalvez J, Enciso M, et al. Simple determination of human sperm
DNA fragmentation with an improved sperm chromatin dispersion test. Fertil Steril. 2005;84(4):833-42, https://doi. org/10.1016/j.fertnstert.2004.11.089.

16. Iorio R, Delle Monache S, Bennato F, Di Bartolomeo C, Scrimaglio R, Cinque B, et al. Involvement of mitochondrial activity in mediating ELF-EMF stimulatory effect on human sperm motility. Bioelectromagnetics. 2011;32(1):15-27, https://doi.org/10.1002/bem.20602.

17. Iorio R, Scrimaglio R, Rantucci E, Delle Monache S, Di Gaetano A, Finetti N, et al. A preliminary study of oscillating electromagnetic field effects on human spermatozoon motility. Bioelectromagnetics. 2007;(28):72-5, https://doi. org/10.1002/bem.20278.

18. Roychoudhury S, Jedlicka J, Parkanyi V, Rafay J, Ondruska L, Massanyi P, et al. Influence of a $50 \mathrm{~Hz}$ extra low frequency electromagnetic field on spermatozoa motility and fertilization rates in rabbits. J Environ Sci Health A Tox Hazard Subst Environ Eng. 2015;44(10):1041-7, https://doi. org/10.1080/10934520902997029.

19. Falahati SA, Anvari M, Khalili MA. Effect of combined magnetic fields on human sperm parameters. Iran J Radiat Res. 2011;9(3):195-200.

20. Łopucki M, Jakiel G, Bakalczuk S, Pietruszewski M, Kankofer J. Influence of alternating magnetic field with magnetic induction $0.5 \mathrm{mT}$ and frequency $50 \mathrm{~Hz}$ on human spermatozoa in-vitro. Int J Androl. 2005;28 Suppl 1:45-129.

21. Formicki K, Szulc J, Tański A, Korzelecka-Orkisz A, Witkowski A, Kwiatkowski P. The effect of static magnetic field on Danube huchen, Hucho hucho (L.) sperm motility parameters. Arch Pol Fish. 2013;21:189-97, https://doi.org/ 10.2478/aopf-2013-0016.

22. Xu X, Lin H, Zhang X, Li J, Zhang W, Sun W, et al. [The effects of extremely low frequency electromagnetic field exposure on the $\mathrm{pH}$ of the adult male semen and the motoricity parameters of spermatozoa in vitro]. Zhonghua Lao Dong Wei Sheng Zhi Ye Bing Za Zhi. 2012;30(3):178-80. Chinese.

23. Bernabò N, Tettamanti E, Russo V, Martelli A, Turriani M, Mattoli M, et al. Extremely low frequency electromagnetic 
field exposure affects fertilization outcome in swine animal model.Theriogenology.2010;73(9):1293-305,https://doi.org/ 10.1016/j.theriogenology.2009.12.010.

24. Bernabò N, Tettamanti E, Pistilli MG, Nardinocchi D, Berardinelli P, Mattioli M, et al. Effects of $50 \mathrm{~Hz}$ extremely low frequency magnetic field on the morphology and function of boar spermatozoa capacitated in vitro. Theriogenology. 2007;67(4): 801-15, https://doi.org/10.1016/j.theriogenology.2006.10.014.

25. Wdowiak A, Wdowiak L, Wiktor H. Evaluation of the effect of using mobile phones on male fertility. Ann Agric Environ Med. 2007;14(1):169-72.

26. Adams JA, Galloway TS, Mondal D, Esteves SC, Mathews F. Effect of mobile telephones on sperm quality: A systematic review and meta-analysis. Environ Int. 2014;70:106-12, https://doi.org/10.1016/j.envint.2014.04.015.

27. Liu C, Yu J, Yang Y, Tang X, Zhao D, Zhao W, et al. Effect of $1 \mathrm{mT}$ sinusoidal electromagnetic fields on proliferation and osteogenic differentiation of rat bone marrow mesenchymal stromal cells. Bioelectromagnetics. 2013;34(6):45364, https://doi.org/10.1002/bem.21791.

28. Avendano C, Mata A, Sanchez Sarmiento CA, Doncel GF. Use of laptop computers connected to internet through WiFi decreases human sperm motility and increases sperm DNA fragmentation. Fertil Steril. 2012;97(1):39-45.e2, https://doi.org/10.1016/j.fertnstert.2011.10.012.

29. Freour T, Barriere P. Wi-Fi decreases human sperm motility and increases sperm DNA fragmentation. Fertil Steril. 2012;97(4):e14; author reply e15, https://doi.org/10.1016/ j.fertnstert.2012.02.004.

30. Yildirim ME, Kaynar M, Badem H, Cavis M, Karatas OF, Cimentepe E. What is harmful for male fertility: Cell phone or the wireless Internet? Kaohsiung J Med Sci. 2015;31(9): 480-4, https://doi.org/10.1016/j.kjms.2015.06.006.

31.Zmyślony M, Politański P. [The risk assessment and safety measures for workers exposed to electromagnetic radiation 0-300 GHz]. Łódź: Instytut Medycyny Pracy; 2009. Polish.

32. O'Flaherty C. Redox regulation of mammalian sperm capacitation. Asian J Androl. 2015;17(4):583-90, https://doi. org/10.4103/1008-682X.153303.

33. Correia J, Michelangeli F, Publicover S. Regulation and roles of $\mathrm{Ca}^{2+}$ stores in human sperm. Reproduction. 2015;150(2):R65-76, https://doi.org/10.1530/REP-15-0102.

34. Walleczek J. Electromagnetic field effects on cells of the immune system: The role of calcium signaling. FASEB J. 1992; 6(13):3177-85, https://doi.org/10.1096/fasebj.6.13.1397839.

35. Lim J-H, McCullen SD, Piedrahita JA, Loboa EG, Olby NJ. Alternating current electric fields of varying frequencies: Effects on proliferation and differentiation of porcine neural progenitor cells. Cell Reprogram. 2013;15(5):405-12, https://doi.org/10.1089/cell.2013.0001.

36. Liu K, Li Y, Zhang G, Liu J, Cao J, Ao L, et al. Association between mobile phone use and semen quality: A systemic review and meta-analysis. Andrology. 2014;2(4):491-501, https://doi.org/10.1111/j.2047-2927.2014.00205.x.

37. Wang C, Leung A, Tsoi WL, Leung J, Ng V, Lee KF, et al. Evaluation of human sperm hyperactivated motility and its relationship with the zona-free hamster oocyte sperm penetration assay. J Androl. 1991;12:253-7, https://doi.org/ 10.1002/j.1939-4640.1991.tb00264.x.

38. Holt W, Watson P, Curry M, Holt C. Reproducibility of computer-aided semen analysis: Comparison of 5 different systems used in a practical workshop. Fertil Steril. 1994;(62):1277-82.

39. Verstegen J, Iguer-Ouada M, Onclin K. Computer assisted semen analyzers in andrology research and veterinary practice. Theriogenology. 2002;57(1):149-79, https://doi.org/ 10.1016/S0093-691X(01)00664-1.

40. Wdowiak A. Sperm epigenetic profile and risk of cancer. J Pre-Clin Clin Res. 2014;8(2):67-70.

This work is available in Open Access model and licensed under a Creative Commons Attribution-NonCommercial 3.0 Poland License - http://creativecommons.org/ licenses/by-nc/3.0/pl/deed.en. 